\title{
Warfarin Management Pathway: A clear and safe algorithm, from admission to discharge.
}

\author{
Alice Hart-George
}

NHS

\begin{abstract}
Warfarin is the most commonly prescribed anticoagulant in the UK and the one most frequently associated with both fatal medication errors and litigation claims [1]. Its life-threatening interactions and side effects are a concern for all doctors. Identifying and implementing solutions to achieve safer prescribing and monitoring is imperative to improve patient safety. The National Patient Safety Agency (NPSA) has outlined the major risks associated with anticoagulant therapy and sought to establish safer practice [1]. The monitoring of safety indicators has been highlighted as a solution. This quality improvement project (QIP) introduces a management algorithm for oral anticoagulant therapy in hospital patients, validated through a completed audit cycle. It was completed at one district general hospital (DGH) in England and involved all inpatient wards.
\end{abstract}

Doctors and pharmacists were interviewed to assess their knowledge of the correct pathways for management of patients on warfarin. The number of errors on hospital warfarin charts was audited over three weeks. These results, coupled with senior haematological advice led to the production of an algorithm illustrating the gold-standard pathway for warfarin management from admission to discharge. It was emailed to all doctors in the Trust and a laminated copy attached to hospital Pneumatic Tube System (PTS) machines. The warfarin charts were reaudited over the following three weeks. The results showed a marked decrease in errors and incomplete anticoagulation referrals as well as a reduction in doctors' anxiety around prescribing warfarin.

\section{Problem}

Understanding warfarin management pathways can be challenging, especially for new doctors and trainees moving hospitals on a yearly basis. With no national drug chart and local variations in approach to follow up, management is open to confusion and error.

In the DGH concerned, this was found to be the case, with omissions and errors on the warfarin prescription charts (which doubled as referrals to the anticoagulation clinic) occurring on a daily basis. At this hospital, warfarin was not prescribed on the standard drug chart, but on a separate booklet of pink paper known as a 'warfarin chart' or 'pink form'. Having all sections of this filled out was considered the 'gold standard' in documentation for warfarin prescribing and referral to anticoagulation clinic.

A number of clinical incidents had been highlighted that had compromised patient safety, for example, patients being discharged on warfarin without an appointment to get their International Normalised Ratio (INR) checked. Such incidents had illustrated the serious failures of the current system and its need to be addressed. The lead biomedical scientist for the anticoagulation clinic remarked in interview, "It is a terrible problem, incomplete or inaccurate warfarin prescriptions are received on a daily basis".

The QIP was carried out in the haematology and biomedical science/anticoagulation departments. Its primary outcome was to find a way to help doctors meet the clinical standards expected of them.
The front page of the warfarin chart had ten information sections to be filled out:

1. Patient details: name, hospital number, date of birth, address on discharge, telephone number, GP.

2. Ward, consultant, completed by, bleep no.

3. Indication for anticoagulation

4. Desired INR range

5. Desired duration

6. Underlying medical conditions

7. Discharge medications

8. If aspirin/clopidogrel/dalteparin to continue on discharge, creatinine, thrombophilia screen.

9. Date of booked clinic appointment or advised next INR blood test.

10. Date patient counselled and by whom.

Boxes on the back were used for the prescribing of loading and maintenance doses. There were general management guidelines on the inside of the paper, but in a block of text that took time and effort to understand.

The anticoagulation team had been struggling with doctors not completing warfarin charts correctly and had devised a rejection letter entitled, 'Notice of rejection of incomplete referral to anticoagulation clinic', to little effect. They felt they were wasting their time chasing doctors for complete forms, or at worst, rectifying situations that occurred from the poor management of patients.

There had been no formal teaching to explain how to manage 
patients on warfarin (beyond dosing and INR). This had led to uncertainty and anxiety amongst new doctors, as revealed by the preliminary interviews. The lack of a clear, easy to understand, management pathway was thought to be a main reason behind poor documentation. Other reasons included time pressures when filling in the forms, too many sections and a lack of clarity regarding when the form should be completed and by whom. There was a need to improve patient safety through the introduction of clearly presented guidelines to address these uncertainties.

\section{Background}

Most published literature on warfarin relates to pathological consequences rather than quality of care delivered [2]. Nevertheless, anticoagulants were included in the Department of Health Report Making Medication Practice Safer (2004) [3] as highrisk medicines that require the implementation of additional safety controls. As a consequence, the British Committee for Standards in Haematology (BCSH) and National Patient Safety Agency (NPSA) [1], published a risk assessment of anticoagulant therapy in order to reduce the risks associated with anticoagulant use and to set standards for service development and controls assurance [4]. This assessment included fifteen points, of which these outlined below had particular relevance to the audit:

Inadequate competencies and training of staff undertaking anticoagulant duties.

Unsafe arrangements and communication at discharge from hospital including failure to adequately transfer duty of care to patient's general practitioner.

Poor documentation of reason and treatment plan at commencement of therapy.

Unconsidered co-prescribing and monitoring of interacting drugs.

\section{1: Failure in training and competence:}

There was no induction training for foundation year or new doctors on the idiosyncratic nature of warfarin management in the DGH. Junior doctors reported anxiety around prescribing warfarin with one stating in a pre-intervention interview, "I'm not sure when to send the form to the anticoagulation clinic". There was no formal system of clinical supervision for senior staff to oversee and assess the competence of new doctors' prescribing.

\section{2: Failure in procedures and clinical protocol:}

Although there was a written clinical protocol, it was in a block of A4 text on the inside of the warfarin charts, making it hard to interpret. There were no effective communication systems to outline the transferring of clinical responsibility for anticoagulant therapy e.g. on discharge from hospital. This led to failure in care transitions, exacerbated by the use of paper 'to take home' prescriptions (TTO's). The anticoagulation team had to spend time chasing doctors to complete referrals, finding patients whose details were not documented and cancelling anticoagulation appointments for inappropriate referrals.

3: Poor documentation:

Patients were often not issued with patient-held information and written dosage instructions at start of therapy, or discharged from hospital without an appointment for next INR measurement or for consultation with appropriate healthcare professional. New referrals to anticoagulation service had incomplete information making appointments more challenging for the haematology team. Patients were affected by reduced quality of anticoagulation leading to potentially life-threatening over or under coagulation.

4: Failure to consider significant pharmacological interactions:

Not enough safe practice had been promoted regarding coprescribing one or more clinically significant interacting medicines or informing anticoagulant services that an interacting medicine had been prescribed. Co-prescribed medications were frequently not documented on the referral form, with the consequence that the anticoagulation team were unable to identify any potentially dangerous interactions in a timely manner.

\section{Baseline Measurement}

Doctors and pharmacists were interviewed to assess their knowledge of the correct pathways for management of patients on warfarin and allow them to highlight any concerns. Haematology and biomedical teams were interviewed to assess the scale of the problem. After the QIP had been introduced they were reinterviewed. This provided qualitative data.

The number of errors on completed hospital warfarin prescriptions was prospectively audited over three weeks, providing quantitative data. All incomplete forms were photocopied and put to one side for analysis. Inappropriate or missed referrals to the anticoagulation on clinic were recorded. Dosing errors were deemed outside the scope of the study.

Over three weeks, $53 \%$ of warfarin prescriptions (24/45) sent to the anticoagulation clinic had major omissions. $67 \%$ of those (16/24) had more than 5 (out of 10 ) sections left totally blank. $42 \%$ (19/45) had no date for the next INR appointment, 42\% (19/45) also had no contact telephone number. 27\% (12/45) had no desired duration. One prescription had nothing but a hospital sticker on it; no contact number, ward or consultant, no prescriber name, no indication, INR range, duration, concurrent medications, underlying medical conditions or future INR test day. Out of the 24 forms with major omissions 9 were from 3 individuals. The department with the most omissions was the Acute Medical Unit (AMU) with 25\% (6/24). There was one missed referral to the anticoagulation clinic, described in the 'case example' below.

\section{CASE EXAMPLE}

An 87-year-old female, Mrs BA was admitted with shortness of breath. Diagnosed with bilateral pulmonary emboli, she was treated with dalteparin and started on warfarin. During her admission, her 
INR was consistently below range so on discharge she was continued on daily dalteparin injections as well as warfarin. She was given a yellow book and booked into the anticoagulation clinic for the following week but she was not informed about the appointment nor was it documented it in her yellow book. Her warfarin prescription was incomplete with no discharge address, telephone number or concurrent medications. When she failed to attend her appointment, the anticoagulation team were unable to contact her due to this lack of information. After contacting her GP, it was discovered that she had gone to stay with her granddaughter for two weeks post discharge. A district nurse was urgently arranged to collect an INR, which came back as 12.2. She had to receive emergency vitamin $\mathrm{K}$ in the community and be re-booked into the next anticoagulation clinic.

See supplementary file: ds2112.jpg - "Pre intervention results and Warfarin Management Pathway Intervention"

\section{Design}

The baseline results, coupled with advice from a consultant haematologist, led to the production of a flow chart illustrating the correct pathway for warfarin management from admission to discharge, including differences in management between those already on warfarin and those new to it. It directly addressed problems highlighted by the QIP, emphasising the importance of completing the warfarin chart in full. It was also presented in an easy-to-read format, rather than an A4 chunk of text. On the reverse of the flow chart common drugs, foods and herbal preparations that have interactions with warfarin were listed e.g. enzyme inducers and inhibitors. The algorithm and an accompanying letter of explanation were sent to all doctors in the Trust, copies were printed and put up in wards and handover rooms, laminated and stuck to PTS machines. Two weeks after distribution a re-audit took place and the impact was assessed.

\section{Strategy}

Problem areas were highlighted in the interviews, particularly regarding the lack of knowledge of the clinical protocol. Many mentioned the ill-defined roles surrounding taking responsibility for full completion of the chart e.g. the person starting the chart or the person discharging the patient. There was a heavy reliance on the anticoagulation team or GPs to make necessary appointments for INR checks rather than, as should have been the case, the discharging doctors arranging these.

Improvement (PDSA) Cycle 1

The design of the algorithm was discussed with consultants, registrars and junior doctors in general medicine and haematology, along with the lead biomedical scientist and other members of the multidisciplinary team working in the anticoagulation clinic.

Improvement (PDSA) Cycle 2

The comments received in the feedback from cycle 1 were taken into account and implemented. The warfarin algorithm was posted up in the doctors mess and feedback welcomed. An example of feedback changing design was the colour scheme of using the same pink paper for the flow chart, as used for the warfarin prescriptions so a clear association between the two was easily achieved. It was also suggested that a copy of the flow chart be given to all new doctors.

\section{Results}

After the intervention, the hospital warfarin prescriptions were prospectively re-audited over three weeks to assess the effect of the intervention. The results showed a dramatic reduction in incomplete charts.

In the three week post-intervention audit, there were no inappropriate or missed clinic referrals. $4 \%$ (2/47) of prescriptions sent to the anticoagulation clinic were found to have omissions. Only one chart had major omissions ( $>5 / 10$ sections blank).

See supplementary file: ds2111.jpg - "Warfarin post intervention results"

\section{Lessons and Limitations}

It has become clear that it is possible to make significant and hopefully, lasting improvements in hospital management and patient safety. Initially, it was a challenge to encourage senior staff to engage in the QIP process but because the services of Doctors Advancing Patient Safety (DAPS) was utilised, a mentor outside of the DGH was found and the project could continue without input from local seniors.

Subsequent to the successful completion of the project, the warfarin management pathway has remained widely available on the medical wards and was well received by junior doctors with noted support from senior members of staff.

Why were the forms filled in so poorly?

- Education: doctors may not be aware of the importance of completing the forms in full. Interviews suggested doctors were unaware of how the anticoagulation services were run.

- Recognition: doctors did not recognise the formality of the warfarin chart as a drug chart in its own right, it being separate and more 'flimsy' pink paper, rather than the standard drug chart.

- Time: doctors are pressed for time and will often only fill in the most basic information that they deem necessary. The form has 10 sections with a total of 20 questions within it. This requires time to complete e.g. checking creatinine, patient weight or discharge medications.

- Responsibility: the doctor who starts the form (often in the medical/surgical admissions unit) is generally not the doctor who discharges the patient. There was no clear assignment of responsibility for completing the form. 
The QIP was cost effective in that its total cost (approximately $£ 34.00$ for paper, photocopying and laminating) was less than the cost of one missed outpatient appointment (approximately £156 [5]), not to mention the legal costs if a serious error were to result in patient harm.

The project was limited by its small sample size, short study period, and re-audit immediately post-intervention. Poor practice could return because of a high turnaround of staff, and so a re-audit one year on is therefore desirable.

It is vital that patient safety remains a priority and hospital practice is constantly reviewed and refined to meet the high standards expected of the NHS. It is everyone's responsibility to be engaged in programmes that work towards improving standards of care.

Implementation of this management algorithm would help standardise management for hospital patients taking anticoagulant drugs, and should reduce morbidity and mortality associated with administration of such medication, from admission, until beyond discharge.

\section{Conclusion}

The warfarin management algorithm is a simple, cheap and effective educational intervention that helps to reduce errors in the management of patients on warfarin. It can be implemented in any setting, altered appropriately for each Trust and can be easily saved on smartphones. Educating healthcare professionals on correct management prevents potentially lethal mistakes, decreases prescribers' anxiety regarding correct management, and saves money and time through reducing inappropriate clinic referrals. Until a national prescribing system is designed and implemented, it is particularly important for hospital staff to pay attention to their idiosyncratic systems and design easily understood and readily available instructions for meeting standards.

\section{References}

1. Cousins D, Harris W. Risk assessment of anticoagulant therapy. [Internet]. London: NHS National Patient Safety Agency; 2006. [cited 2013 Jul 27]. Available from: http://www.nrls.npsa.nhs.uk/EasySiteWeb/getresource.axd? Asset|D $=60022 \& \ldots$

2. Baglin T, Cousins D, Keeling D, Perry D, Watson H. Recommendations from the British Committee for Standards in Haematology and National Patient Safety Agency. Br J Haematol. 2006;136:26-29

3. Smith J. Building a safer NHS for patients, improving medication safety. [Internet]. London; NHS Department of Health; 2004. [cited 2013 Jul 27]. Available from: http://webarchive.nationalarchives.gov.uk/20130107105354/ http://www.dh.gov.uk/prod_consum_dh/groups/dh_digitalass ets/@dh/@en/documents/digitalasset/dh_4084961.pdf

4. Keeling D, Baglin T, Tait C, Watson H, Perry D, Baglin C. et al. Guidelines on oral anticoagulation with warfarin - fourth edition. Br J Haematol. 2011;154(3):311-324
5. Drfosterhealth.co.uk [Internet]. Outpatient appointment noshows cost hospitals $£ 600 \mathrm{~m}$ a year. London: Dr Foster c2009 [cited 2013 Jul 27]. Available from: http://www.drfosterhealth.co.uk/features/outpatientappointment-no-shows.aspx

\section{Declaration of interests}

Nothing to declare

\section{Acknowledgements}

Imran Qureshi

lain Milligan 